\title{
Gastroösophageale Refluxkrankheit und ihre Komplikationen
}

\author{
Joachim Mössner \\ Medizinische Klinik und Poliklinik für Gastroenterologie und Rheumatologie, Department für Innere Medizin, \\ Neurologie und Dermatologie, Universitätsklinikum Leipzig, AöR, Deutschland
}

\begin{abstract}
Die gastroösophageale Refluxkrankheit (GERD) nimmt in den westlichen Industrienationen an Häufigkeit zu. Ob diese $\mathrm{Zu}$ nahme nur an unseren Ess- und sonstigen Lebensstilgewohnheiten und insbesondere an der Zunahme des Übergewichts liegt, ist letztlich noch nicht abschließend geklärt. Nahezu zeitgleich ist eine deutliche Zunahme des Adenokarzinoms, des sogenannten Barrett-Karzinoms, zu verzeichnen. Es gilt als sicher, dass außer möglichen prädisponierenden genetischen Faktoren der pathologische Magensäure- und wahrscheinlich auch duodeno-gastroösophageale Reflux mit Gallensäuren und Pankreasenzymen in der Pathogenese der Entstehung der intestinalen Metaplasie im Ösophagus eine Rolle spielt. Diese Schleimhaut ist eine Präkanzerose. Gleichzeitig schützt sie aber auch vor der Säure. Dies mag erklären, dass viele Patienten mit Barrett-Karzinom letztlich nicht über eine langjährige vorbestehende Symptomatik mit Refluxsymptomen klagen. Andererseits hat eine schwedische Studie [1] klar gezeigt, dass häufige Refluxsymptome das Risiko des Auftretens eines Barrett-Karzinoms erhöhen. Bei regelmäßig auftretenden Refluxsymptomen wird daher eine sogenannte Index-Endoskopie empfohlen. Bei Nachweis einer Barrett-Schleimhaut werden dann regelmäßig endoskopische Verlaufskontrollen empfohlen. Trotz zahlreicher, äußerst innovativer endoskopischer Technologien, siehe Artikel von Rey, Labenz, Hansen und Kiesslich [2] aus Mainz und Siegen, bleibt die Frühdiagnose eines Karzinoms bei bekanntem Barrett-Epithel ein bisher unbefriedigend gelöstes Problem. Diese Nachsorge konnte bislang nicht belegen, dass die Letalität am Barrett-Karzinom gesenkt wird. Wie sollen wir dann erst recht bei den Patienten ein Barrett-Karzinom rechtzeitig entdecken, die vorher keine Refluxsymptomatik aufweisen? Vom Ziel der Prophylaxe oder Frühdiagnose, wie wir es wahrscheinlich beim kolorektalen Karzinom erreichen, sind wir leider noch weit entfernt.
\end{abstract}

Aufgrund ihrer beeindruckenden Wirksamkeit bezogen auf Säuresuppression und Symptombefreiung bei erosiver Refluxösophagitis werden Protonenpumpeninhibitoren (PPI) sehr großzügig eingesetzt, zumal auch ihr Nebenwirkungspotenzial erstaunlich niedrig ist. Doch nicht nur bei Nichtansprechen der me- dikamentösen Therapie, sondern generell sollten andere Ösophaguserkrankungen nicht vergessen werden. In der Arbeit von Miehlke [3] aus Hamburg werden diese Erkrankungen vorgestellt.

Bei der Häufigkeit der Refluxkrankheit wird auch vermutet, dass Säurereflux über nervale Reflexbögen und/oder Mikroaspirationen für Asthma bei einigen Patienten verantwortlich sein kann. Säurereflux wird als Ursache von chronischer Heiserkeit auf dem Boden einer Laryngitis, von Zahnschmelzerosionen usw. angeschuldigt. Im Artikel von Madisch aus Hannover und Labenz aus Siegen [4] wird die Literatur zu diesem Thema kritisch dargestellt.

Sprechen Patienten auf PPI nicht an oder sollen Patienten unter der Annahme einer Refluxkrankheit operiert werden, und sind die Symptome ferner untypisch, ist eine Ösophagusfunktionsdiagnostik unverzichtbar. Im Artikel von Leodolter [5] werden diese diagnostischen Möglichkeiten dargestellt. Wie bereits erwähnt, sind PPI in der Therapie der Refluxkrankheit unverzichtbar geworden. Allein in Deutschland sind die Umsatzzahlen gewaltig: 2009 fast 2 Milliarden definierte tägliche Dosierungen (DDDs) [6]. Der vermehrte klinische Einsatz von PPI reflektiert einerseits die Wirksamkeit dieser Substanzen bei der Ulkuskrankheit, der Refluxkrankheit und der Prävention und Therapie von Erosionen und Ulzerationen, die unter der Einnahme von nicht steroidalen Antirheumatika im Magen und Duodenum entstehen; andererseits nimmt die Refluxkrankheit in den Industrienationen sicher nicht so zu, um diesen weiteren Anstieg der Verordnung von PPI zu erklären, zumal ja auch das durch Helicobacter pylori verursachte Ulkusleiden in den gleichen Ländern immer seltener vorkommt. PPI werden wahrscheinlich bei dem sehr häufigen Krankheitsbild der «funktionellen Dyspepsie», dem Reizmagen-Syndrom, eingesetzt, obgleich für diese Indikation die wissenschaftliche Evidenz kaum belegt ist. Im Artikel von Labenz aus Siegen und Kiesslich aus Mainz [7] werden die konservativen Therapiemöglichkeiten der Refluxerkrankung, unter Wertung der Literatur und evidenzbasiert, sehr kritisch dargestellt. Die bessere Wirksamkeit der PPI bei erosiver Reflux-

\section{KARGER \\ Fax +497614520714 \\ Information@Karger.de}

www.karger.com (c) 2011 S. Karger GmbH, Freiburg

$1662-6664 / 11 / 0272-0103 \$ 38.00 / 0$

Accessible online at:

www.karger.com/vim
Prof. Dr. med. Joachim Mössner

Medizinische Klinik und Poliklinik für Gastroenterologie und Rheumatologie

Department für Innere Medizin, Neurologie und Dermatologie, Universitätsklinikum Leipzig, AöR

Liebigstraße 20, 04103 Leipzig, Deutschland

Tel. +49 341 97-12200

Joachim.Moessner@medizin.uni-leipzig.de 
erkrankung im Vergleich zur NERD (Non Erosive Reflux Disease) muss daran denken lassen, dass bei vielen NERD-Patienten auch andere Pathomechanismen als «nur» Säurereflux eine Rolle für ihre Beschwerden spielen. Dies wird auch belegt durch die Tatsache, dass gerade NERD-Patienten nicht von einer Fundoplicatio profitieren.

Viele unserer heutigen kritischen Patienten stehen auch einer medikamentösen Dauertherapie kritisch gegenüber. In Anbetracht möglicher Risiken einer Operation ist daher die Idee, endoskopisch die Refluxbarriere am ösophagogastralen Übergang wiederherzustellen, genial. Viele der endoskopischen Verfahren, die teils auch unkritisch von der US-amerikanischen Gesundheitsbehörde (FDA) zugelassen wurden, hielten nicht, was sie versprachen. Mein ehemaliger Mitarbeiter Caca war maßgeblich an etlichen Studien zu diesem Thema beteiligt. Im Artikel von Cahyadi und Caca [8] aus Ludwigsburg werden diese endoskopischen Verfahren dargestellt. Da bei den derzeit noch verfügbaren Methoden nur schlanke Patienten mit kleinen Hernien profitieren, kommen endoskopische Verfahren für die Mehrzahl der Patienten mit Refluxerkrankung nicht infrage. Fuchs, Breithaupt, Varga und Schulz [9] aus Frankfurt/M. stellen als Chirurgen sehr «selbstkritisch» die Vor- und Nachteile der laparoskopischen Fundoplicatio mit ihren technischen Variationen gegenüber einer PPI-Langzeit-Therapie dar. Ich bin überzeugt, dass es zwischen Internisten/Gastroenterologen und in der laparoskopischen Fundoplicatio erfahrenen Viszeralchirurgen keine wirkliche Kontroverse mehr gibt, welcher Patient dauerhaft medikamentös konservativ behandelt werden sollte und welcher Patientengruppe die genannte Operation angeboten werden sollte.

Faszinierend sind zweifelsohne die endoskopischen Möglichkeiten, mittels Mukosaresektion maligne Neoplasien des Ösophagus zu behandeln, die auf die Mukosa begrenzt sind und histologisch keine Risikokriterien haben, bereits in die Lymphknoten metastasiert zu sein. Hier hat die Arbeitsgruppe um Ell aus Wiesbaden durch Studien international anerkannte Kriterien erarbeitet, welche Patienten für diese Form der Therapie geeignet sind. Der Pathologe hat gewissermaßen das «letzte Wort». Er kann nach Mukosaresektion feststellen, ob eine R0Resektion vorliegt (siehe Artikel von May [10] aus Wiesbaden).
Die positiven Kurz- und Langzeitergebnisse bezüglich Komplikationsrate, Auftreten einer Lymphknotenmetastasierung, rezidivfreiem Überleben und Gesamtüberleben haben den endoskopischen Verfahren einen festen Platz in der multimodalen Therapie des Ösophaguskarzinoms eingeräumt. Weitere Studien müssen zeigen, ob dieses Verfahren auch für Karzinome, die nur oberflächlich die Submukosa infiltriert haben, geeignet ist, wenn die histologischen Kriterien des Tumors dies erlauben, d.h. das Risiko einer bereits vorliegenden Lymphknotenmetastasierung sehr gering ist. Dieser Punkt, wann die Operation bei primär operablen Patienten unabdingbar ist, wird vielleicht zwischen in der Mukosaresektion erfahrenen Gastroenterologen und Viszeralchirurgen noch kontrovers diskutiert. Aber auch diese Frage wird durch Studien beantwortet werden. Es darf besonders betont werden, dass diese Patienten nur in «High Volume»-Zentren interdisziplinär behandelt werden sollten, die auch über das gesamte Repertoire einer exzellenten Diagnostik (siehe [2]) verfügen und insbesondere in der endosonografischen Diagnostik zum Nachweis der lokalen Lymphknotensituation sehr erfahren sind. Die chirurgische Arbeitsgruppe von Hölscher [11] aus Köln zeigt State of the Art, welche Operationsverfahren mit ihren jeweiligen Vor- und Nachteilen zur Behandlung des Barrett-Karzinoms zur Verfügung stehen und mit welchen Komplikationen und Langzeitergebnissen zu rechnen ist. In dieser Arbeit wird auch der Stellenwert der neoadjuvanten Radiochemotherapie behandelt und die noch unzureichende Datenlage des Stellenwerts der adjuvanten Chemotherapie beschrieben.

Das interdisziplinäre Gespräch ist in der Regel ein fester und beliebter Bestandteil unserer Zeitschrift VISzERALMEdizIN. Wie in diesem Editorial hervorgehoben, ist die Diagnostik und Therapie der Refluxkrankheit und ihrer Komplikationen so komplex, dass wir uns nicht auf einen Teilaspekt in Form des interdisziplinären Gesprächs reduzieren wollten. Ich bitte daher um das Verständnis der Leser, dass diese Rubrik im Heft fehlt. Ich würde mich als Herausgeber dieser Ausgabe der Viszeralmedizin sehr freuen, wenn die Artikel das Interesse unserer Leser finden würden. Ich freue mich, dass führende Experten zum Thema Refluxkrankheit den aktuellsten Stand ihrer eigenen Erfahrungen im Kontext mit der Weltliteratur präsentieren.

\section{Literatur}

1 Lagergren J, Bergström R, Lindgren A, Nyrén O: Symptomatic gastroesophageal reflux as a risk factor for esophageal adenocarcinoma. N Engl J Med 1999;340:825-831.

2 Rey JW, Labenz J, Hansen T, Kiesslich R: Gastroösophageale Refluxkrankheit und ihre Komplikationen - die endoskopische Diagnostik. Viszeralmedizin 2011;27(2):133-140.

3 Miehlke S: Differentialdiagnose Ösophagitis: Eosinophile Ösophagitis, Candida, Cytomegalievirus. Viszeralmedizin 2011;27(2):121-125.

4 Madisch A, Labenz J: Extraösophageale Manifestationen der Refluxkrankheit: Fakt oder Fiktion? Viszeralmedizin 2011;27(2):146-151.
5 Leodolter A: Funktionsdiagnostik bei gastroösophagealer Refluxkrankheit - von der Manometrie über BRAVO und Langzeit-pH-Metrie zur Impedanzmessung. Viszeralmedizin 2011;27(2):141-145. 6 Mössner J: Magen-Darm-Mittel und Laxantien; in Schwabe U, Paffrath D (Hrsg): Arzneiverordnungsreport 2010; Kapitel 32. Berlin, Springer, 2010, pp 653-681.

7 Labenz J, Kiesslich R: Konservative Therapie: Lebenswandeländerung, Antazida, $\mathrm{H}_{2}$-Blocker oder doch nur Protonenpumpeninhibitoren? Viszeralmedizin 2011;27(2):113-119.

8 Cahyadi O, Caca K: Endoskopische Antirefluxtherapie - was bleibt übrig? Viszeralmedizin 2011; 27(2):106-111.
9 Fuchs KH, Breithaupt W, Varga G, Schulz T: Laparoskopische Fundoplicatio: Lösung des Problems? Viszeralmedizin 2011;27(2):127-132.

10 May A: Endoskopische Therapie bei Barrett-Neoplasie: Endoskopische Resektion, Radiofrequenzablation, photodynamische Therapie - wann welches Verfahren? Viszeralmedizin 2011;27(2): 152-156.

11 Vallböhmer D, Schröder W, Brabender J, Hölscher AH: Chirurgie des Barrett-Adenokarzinoms. Viszeralmedizin 2011;27(2):157-161. 